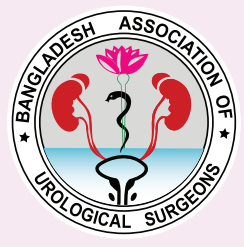

\title{
Non Muscle-Invasive Bladder Cancer (NMIBC)
}

\author{
MA Salam
}

\section{Introduction}

Carcinoma of the urinary bladder is the $9^{\text {th }}$ most common malignancy in the world, and urothelial carcinoma accounting for $90 \%$ of all histological subtypes. ${ }^{1}$ NMIBC or Non muscle-invasive bladder cancer is a heterogeneous sub classification of urothelial carcinoma with significant variation in individual risk of recurrence and progression to muscle-invasive disease. Risk stratification by American Urological Nomograms/risk calculators developed from clinical trial data by Association (AUA) and European Association of Urology (EAU) can help inform patient treatment decisions but may not accurately classify all patients. ${ }^{2}$

Non muscle-invasive bladder cancer (NMIBC) accounts for about $70 \%$ of all bladder cancer and is associated with a $>88 \%$ survival rate over 5 years. However, up to $70 \%$ of NMIBC recur after initial treatment, of which $10 \%-20 \%$ can progress to MIBC. +3 Risk stratification tables of European Organization for Research and Treatment of Cancer (EORTC) patients with high-risk NMIBC (T1 with high grade/G3 or carcinoma in situ) represent a challenging group with an increased 5-year risk of recurrence (up to $80 \%$ ) and progression (up to $50 \%$ ). ${ }^{4}$

Use of adjuvant intravesical instillation with various chemotherapy agents e.g. Mitomycin and Bacillus Calmette-Guérin (BCG) is well studied and associated with excellent outcomes for most patients. However, upwards of $40 \%$ of patients recur within 2 years and roughly $10 \%$ progress to muscle-invasive bladder cancer. Reduction of the treatment burden associated with NMIBC is increasingly needed.

The high rates of recurrence and progression with current therapies for NMIBC necessitate lifelong active surveillance, making bladder cancer the most expensive cancer to treat from diagnosis to death. This fact is driving the need for the development of new treatment in patients with NMIBC.
Intravesical BCG is commonly used as an adjuvant treatment after TURBT for intermediate-high-risk NMIBC. ${ }^{6}$ The local and systemic side effects are common with BCG and can lead to discontinuation of therapy in up to $20 \%$ of patients. ${ }^{7}$ It is important to note that up to $50 \%$ of patients fail BCG, significantly increasing the risk of progression and death. ${ }^{8}$ The patients who have failed BCG therapy may require radical cystectomy with urinary diversion and are also associated with considerable morbidity. The current therapies for NMIBC are associated with high rates of progression and recurrence and side effects. The Radical Cystectomy and Urinary diversion are also associated with considerable side effects and morbidity. There is an urgent need for novel agents in the treatment of NMIBC. ${ }^{5}$

\section{Evaluation of NMIBC}

To establish an accurate diagnosis is critical to the proper treatment of bladder cancer. Diagnosis begins with a detailed history focusing on antecedent symptomatology, comorbidities, risk factors, and family history. Attention to constitutional symptoms and a focused physical examination may uncover findings suggestive of advanced disease. Proper Counseling patients on smoking cessation upon diagnosis can reduce the risk of perioperative complications and may reduce the risk of NMIBC recurrence. ${ }^{89}$

The office-based flexible cystoscopy visually confirms the presence of bladder cancer, which is followed by a transurethral resection of the bladder tumor (TURBT) under anesthesia. Care should be taken during the TURBT, description of tumor size, location, multiplicity, appearance, and associated mucosal changes is essential to staging. Adequate resection of all visible tumor with safe sampling of the underlying detrusor muscle layer should be the goal of all TURBT. ${ }^{90}$

Professor of Urology \& Founder Chairman, UTFB, Dhaka

Correspondence: Prof. MA Salam, Professor of Urooncology \& Founder Chairman, UTFB, Dhaka, E-mail: masalamurology@yahoo.com 
This protocol will be associated with improved histopathologic accuracy and improved oncologic outcomes. When indicated a re-TURBT is not only diagnostic, but also carries prognostic, and therapeutic benefits to patients and it is generally indicated for all high-grade tumors and especially for any initial pathology specimen devoid of detrusor muscle layer. ${ }^{90,91}$

Existence of metastatic or synchronous upper tract disease is evaluated using conventional cross-sectional imaging with delayed drainage films. The CT urogram or MR Urography is the gold standard of evaluating the upper tracts concomitantly with the abdominal and pelvic viscera and lymph nodes. Imaging of Chest with chest X-ray or CT Scan is also required to exclude metastatic disease in the lungs and is particularly important among smokers where a second primary malignancy may be present. $.00,91$

\section{Advanced cystoscopy}

Use of fluorescence cystoscopy with either 5aminolaevulinic acid (5-ALA) or hexaminolevulinic acid-blue light cystoscopy (Cysview ${ }^{\circledR}$, Photocure, Inc., Princeton, NJ; HAL-BLC) is increasingly being adopted. When compared with traditional white light cystoscopy (WLC), fluorescence cystoscopy has demonstrated improved sensitivity in detecting carcinoma in situ (CIS) and is able to detect additional primary tumors missed by WLC in roughly $20 \%$ of patients. ${ }^{92}$

The meta-analyses have also demonstrated improvements in recurrence but not progression or mortality rates when comparing hexamino levulinic acid-blue light cystoscopy (HAL-BLC) with white light cystoscopy (WLC [93-96]. The higher rates of falsepositive results with blue light cystoscopy (BLC) can lower specificity, whereas false-positive rates seen with traditional white light cystoscopy (WLC) have not been considerably different, historically [96]. The Narrowband imaging is another enhanced cystoscopy technique to help improve the visual contrast between normal mucosal surfaces and tissue that is "hyper vascular" such as cancer, but evidence of effectiveness is limited. ${ }^{97}$

The urine cytology is a helpful adjunct to tumor visualization and biopsy because some CIS is difficult to visualize with standard WLC. The urine cytology is quite sensitive to high-grade tumors like CIS but performs worse with low-grade tumors and use is discouraged among those with low-risk NMIBC and normal cystoscopy [98]. The urinary biomarkers continue to be studied as diagnostic adjuncts and as potential alternatives to cystoscopy for either bladder cancer diagnosis or surveillance. ${ }^{99}$

US Food and Drug Administration (FDA), currently approved six urinary biomarkers including those which assess nuclear matrix proteins (NMP22® Bladder Chek ${ }^{\circledR}$ Test, Abbott, Abbott Park, IL), bladder tumor antigen (BTA STAT \& TRAK tests, Polymedco, Cortlandt Manor, NY), and those that utilize fluorescence in situ hybridization (UroVysion Bladder Cancer Kit [UroVysion Kit], Abbott) and fluorescent immunohistochemistry (ImmunoCyt/uCyt+ test, Scimedex, Denville, NJ). With sensitivities ranging from $0.57-0.82$ and specificities between $0.74-0.88$, few have proven accurate enough for routine clinical use in lieu of cystoscopy. ${ }^{100}$

One of the greatest challenges of histopathology to the diagnosis of NMIBC because of the known inter observer variability of pathologic evaluation. It is important that urologist has to provide the pathologists with the best possible specimen devoid of cautery or crush injury. Systematic and complete visual resection with either fractioned or en bloc removal that adequately samples the detrusor muscle is the most important step in the diagnosis and treatment algorithm for NMIBC. The presence or absence of variant histology (e.g., micro papillary, plasmacytoid, sarcomatoid, etc.), as well as factors such as concurrent CIS and lymphovascular invasion are important for risk stratification and may lead to consideration of upfront extirpative treatment with radical cystectomy rather than adjuvant intravesical therapies.

\section{Intravesical instillation of methylene blue}

Intravesical instillation of methylene blue resulted in selective surface staining of bladder tumors in vivo without staining the background of normal urothelium. Staining of human bladder tumors in vivo was accomplished by the intravesical instillation of $0.1 \%$ methylene blue in $0.9 \%$ saline through a Foley catheter under $20 \mathrm{~cm}$ of hydrostatic pressure up to a maximum volume of $400 \mathrm{ml}$.

After 5 minutes contact time, the methylene blue solution was drained, and the bladder was washed with saline. Thereafter, either endoscopic or open surgery was performed. The transitional cell carcinomas in 45 of 48 patients bound methylene blue 
to the surfaces of the tumors but not to normal urothelium.

Higher grade tumors usually bound the dye more extensively than lower grades. The three patients, whose tumors did not bind methylene blue, had received previous chemotherapy, which might account for their being falsely negative. Carcinoma in situ and dysplasia did stain blue.

Areas of hyperplasia and cystitis, however, did not bind methylene blue. In vivo intravesical staining with methylene blue has been a simple and safe procedure which has enhanced the endoscopic localization for biopsy and fulguration/resection of transitional cell carcinomas. ${ }^{101}$

\section{Prevention of Obturator Reflex}

A potential complication of Transurethral Resection of Bladder Tumor (TURBT) is bladder rupture or injury secondary to adductor muscle contraction from obturator nerve stimulation. This risk is increased in resection of lateral wall tumors, as electrosurgical resection of these lesions is more likely to inadvertently stimulate the obturator nerve.

There are several mechanisms for preventing the obturator reflex. Pharmacologic paralysis can reliably inhibit thigh adduction. However, TURBT is often a short procedure that is not amendable to intermediate duration neuromuscular blocking medications. The use of succinylcholine is another option but is limited by its short duration of action, which may not be adequate for the time needed to resect the tumor. Additionally, many patients have contraindications to the use of succinylcholine. Spinal anesthesia does not reliably prevent the obturator reflex.

Regional anesthesia is another potential treatment modality to prevent the obturator reflex during TURBT. Motor blockade of the obturator nerve will prevent this adduction in the event of inadvertent nerve stimulation. Peripheral blockade of the obturator nerve can be combined with either general or spinal anesthesia. Historically, obturator nerve block (ONB) has been considered a technically challenging procedure. Recently, however, successful ultrasound guided ONB techniques have been reported.

Endoscopic injection of lignocaine in to the base of the tumor is an innovative technique to prevent the obturator Jerk. The procedure will involve the injection of $10 \mathrm{ml} 2 \%$ lignocaine through an endoscopic long flexible e.g. Botox needle at the tumor base through a cystoscope. The needle should be advanced from the healthy mucous membrane towards the base of the tumor. Multiple injection may be given as required. This simple procedure can block the obturator nerve branches supplying the area of the field occupying the bladder. The obturator jerk can be eliminated in every single case. ${ }^{102}$

Post surgical adjuvant intravesical treatments after TURBT have demonstrated reduction in the risk of recurrence and progression compared with TURBT alone. Although generally safe, the use of adjuvant intravesical therapy is based on a risk-adapted strategy that aims to balance reduction of recurrence and progression with side effects, especially systemic, from overtreatment. There are several available risk stratification methods using a variety of clinical and pathological factors that can be used to quantify risk of recurrence and progression. Based on each patients' cancer risk profile after TURBT and considered in the context of their comorbidities, a patient-centered discussion about adjuvant treatment options should be undertaken to guide next steps. ${ }^{89}$

\section{Molecular Subtypes of Bladder Cancer}

At the cytogenetic level, NMIBC is genomic stable, usually with a diploid karyotype with few structural genomic rearrangements. It is thought that the loss of chromosome 9 is an early event in the pathogenesis of BC transversal to both NMIBC and MIBC subtypes. MIBCs are frequently aneuploid with several chromosome rearrangements, rendering them genetically unstable. Molecular fingerprint of BC presents alterations in genes from several pathways, mainly mutations in genes of the cell cycle, chromatin regulation, and tyrosine-kinase signaling. ${ }^{113-114}$

Traditionally cystoscopy is still considered the best method for diagnosing UC, but it is invasive, uncomfortable, and can only detect approximately $90 \%$ of lesions. ${ }^{103}$ In addition, when a tumor is discovered and must be biopsied and/or removed, a second procedure is required, transurethral resection of the bladder tumor (TURBT), which requires an anesthesia. Last, the cost of cystoscopy, especially when used to monitor recurrence, is the major reason why perpatient expenses for UC are among the highest for all cancers. ${ }^{104}$

The major problem associated with NMIBC is that after initial TURBT, $50 \%$ to $70 \%$ of patients develop multiple recurrences; $10 \%$ to $20 \%$ of these will progress to MIBC. 
This risk of recurrence and progression calls for lifelong surveillance. The current standard procedure is to perform cystoscopy and evaluate urine cytology every 3 to 4 months in the first 2 years, twice per year in years 3 to 4 , and yearly thereafter. ${ }^{105}$

To date, none have demonstrated sufficient specificity and sensitivity to monitor the general population or replace cystoscopy and cytology in monitoring for recurrence[106]. Urine cytology is particularly insensitive for detecting low-grade tumors. However, advances in genomics have clearly demonstrated that DNA alterations offer great promise for detecting primary or secondary bladder cancer.

NMIBC and MIBC are genetically different [107-110]. NMIBC is characterized by a high frequency of mutations in the FGFR3 oncogene, leading to constitutive activation of the RAS/ MAPK pathway. In MIBC, mutations in the TP53 gene prevail. In general, mutations in FGFR3 and TP53 are mutually exclusive, suggesting that NMIBC and MIBC develop along different oncogenetic pathways. However, these mutations often occur simultaneously in stage pT1 tumors that invade the connective tissue layer underlying the urothelium.

Recently, somatic mutations in the PIK3CAoncogene, which encodes the catalytic subunit p110á of class-IA PI3 kinase, were described in $13 \%$ to $27 \%$ of bladder tumors. ${ }^{111}$ These mutations often coincided with FGFR3 mutations. Mutations in the RAS oncogenes (HRAS, KRAS, and NRAS) have also been found in $13 \%$ of bladder tumors and in all stages and grades; they are mutually exclusive with FGFR3 mutations. Given these findings, analyzing urine sediment for genetic mutations may be a promising strategy for noninvasive detection of bladder cancer.

Due to the lack of disease-specific symptoms, diagnosis and follow-up of bladder cancer has remained a challenge to the urologic community. Cystoscopy, commonly accepted as a gold standard for the detection of bladder cancer, is invasive and relatively expensive, while urine cytology is of limited value specifically in low-grade disease. Over the last decades, numerous molecular assays for the diagnosis of urothelial cancer have been developed and investigated with regard to their clinical use. However, although all of these assays have been shown to have superior sensitivity as compared to urine cytology, none of them has been included in clinical guidelines.

Despite considerable advances in recent years, the authors feel that at this stage the added value of molecular markers for the diagnosis of urothelial tumors has not yet been identified. Current data suggest that some of these markers may have the potential to play a role in screening and surveillance of bladder cancer. Well-designed protocols and prospective, controlled trials will be needed to provide the basis to determine whether integration of molecular markers into clinical decision-making will be of value in the future.

\section{Risk Stratification}

NMIBC is a widely heterogeneous sub classification of bladder cancer and treatment should be approached accordingly. Depending on tumor characteristics, the probability of recurrence for any given patient can range from $15 \%$ to $70 \%$ at 1 year. Several studies and subsequent meta-analyses have proven that adjuvant intravesical treatment after TURBT is an effective means of reducing the risk of recurrence and progression of NMIBC when compared with TURBT alone. ${ }^{120-121}$ Current adjuvant treatment strategies are risk adapted because not all intravesical therapy is similarly effective nor are all treatment options recommended across risk categories.

There are several stratification tools available including nomograms that can be used to estimate individualized risk predictions for recurrence and progression. Post hoc analyses of patient-level data collected during large randomized, controlled trials (RCTs) conducted by the European Organization for Research and Treatment of Cancer (EORTC) and Club Urologico Espanol de Tratamiento Oncologico (CUETO) were used to generate these tools. Both the EORTC and CUETO groups' nomograms and risk models acknowledge that there are unique factors related to an increased risk of recurrence and/ or progression in NMIBC (Table 1). As such, individual risks for recurrence and progression may be calculated from each. These monograms have all been validated and avoid the somewhat arbitrary categorization of patients into broad risk groups. However, there are certain limiting factors with their use that are important to understand and consider. 
Table 1: NMIBC Risk Stratification Groups, Criteria, and Initial Adjuvant Treatment Recommendations From AUA and EAU Guidelines

\begin{tabular}{|c|c|c|c|}
\hline Risk Category & AUA & EUA & Treatment Recommendation \\
\hline Low & $\begin{array}{l}\text { Solitary LG Ta tumor, } \\
<3 \mathrm{~cm} \text { PUNLMP }\end{array}$ & $\begin{array}{l}\text { Solitary LG Ta tumor, } \\
<3 \mathrm{~cm} \text { PUNLMP }\end{array}$ & $\begin{array}{l}\text { Post-TURBT intravesical } \\
\text { chemotherapy }\end{array}$ \\
\hline Intermediate & $\begin{array}{l}\text { Recurrence within } 1 \text { year, LG Ta } \\
\text { Solitary LG Ta, >3 cm } \\
\text { LG Ta, multifocal } \\
\text { HGTa, <3 cm LG Tl }\end{array}$ & $\begin{array}{l}\text { All tumors not defined as } \\
\text { low or high risk }\end{array}$ & $\begin{array}{l}\text { Post-TURBT intravesical } \\
\text { hemotherapy }+ \\
\text { Induction + maintenance BCG for } 1 \text { year } \\
\text { or } \\
\text { Induction + maintenance chemotherapy } \\
\text { for I year }\end{array}$ \\
\hline High & $\begin{array}{l}\text { HG Tl } \\
\text { Any recurrent, HG Ta } \\
\text { HG Ta, >3 cm } \\
\text { Any CIS } \\
\text { Any BCG failure in HG } \\
\text { Any variant histology } \\
\text { Any LVI } \\
\text { Any HG prostatic urethral } \\
\text { involvement }\end{array}$ & $\begin{array}{l}\text { Any Tl } \\
\text { Any HG tumor } \\
\text { Any CIS } \\
\text { Multiple, recurrent, }>3 \mathrm{~cm} \\
\text { tumors* } \\
\text { T1HG with concurrent CIS* } \\
\text { Variant histology } \\
\text { Lymphovascular invasion }\end{array}$ & $\begin{array}{l}\text { Induction } 1 \text { maintenance BCG for } 1-3 \text { years } \\
\text { or } \\
\text { Radical cystectomy }\end{array}$ \\
\hline
\end{tabular}

* Special designation of "highest risk" and upfront radical cystectomy should be considered.

a With an EORTC recurrence score $<5$;

${ }^{\mathrm{b}}$ EAU recommends $1-3$ years, AUA recommends 3 years;

c optimal schedule not known.

AUA, American Urological Association; OS, carcinoma in situ; EAU, European Association of Urology; HG, high grade (G2, all G3); LG, low grade (Gl, some G2); LVI, lymphovascular invasion; NMIBC, non-muscle-invasive bladder cancer; PUNLMP, papillary urothelial neoplasm of low malignant potential, variant histology 5 micropapillary, plasmacytoid, sarcomatoid, etc.

The initial EORTC risk tables were based on patientlevel data from 2596 patients with NMIBC, only $4.4 \%$ of which also had CIS. ${ }^{15}$ In this study, no patients received a second TURBT and $78 \%$ were treated primarily with a variety of intravesical chemotherapy regimens. Only $6.5 \%$ of patients received bacillus Calmette-Guérin (BCG) and none received maintenance treatments. The CUETO group developed a similar nomogram based on data from 1062 patients from 4 randomized trials, all of which were treated with BCG. ${ }^{123}$ Use of maintenance treatment in the CUETO cohort was limited to 5 to 6 months. In 2016, the EORTC subsequently produced a nomogram and additional risk groups specific to a combined cohort of patients from 2 RCTs assessing the benefit of 1 to 3 years of maintenance BCG among 1812 intermediate and high-risk patients, all without CIS. ${ }^{124}$. Risk estimates of early and later recurrence are given based on prior recurrence rate and number of tumors, with tumor grade as an additional prognostic factor for early recurrence. A summary and comparison of risk factors and predicted recurrence and progression rates can be found in (Table) for the original EORTC risk tables and the CUETO nomogram

\section{Types of Adjuvant Therapy}

The adjuvant treatment is any agent administered after complete TURBT aimed at reducing the risks of NMIBC recurrence and progression. Traditionally, only intravesical agents have been used in this setting, but emerging data on the use of systemic checkpoint inhibitor immunotherapies either as a monotherapy or combined with intravesical agents may radically change this treatment paradigm. Current adjuvant intravesical treatments can be defined by the timing and sequence of their administration: (a) preoperatively, usually within $24 \mathrm{~h}$ of TURBT, (b) induction, as an initial course for that particular agent, or (c) maintenance, subsequent courses in the setting of no evidence of disease after TURBT and induction treatment. ${ }^{115}$ 
Standard intravesical therapies can also be categorized as either chemotherapy or immunotherapy agents. The most used contemporary chemotherapy agents are Mitomycin C, gemcitabine, epirubicin, and docetaxel. These are given individually or in combination depending on the indication. Historically, agents such as doxorubicin, valrubicin, cisplatin, and thiotepa, among others, were also frequently studied and used. The gold standard intravesical immunotherapy is BCG, an attenuated mycobacterium strain first described for use in bladder cancer in 1976. Interferon (IFN) has also been studied, but contemporary use is limited. ${ }^{115}$

There are several emerging NMIBC therapies being developed and studied in the treatment-naive or treatment-unresponsive setting. Novel agents like oncolytic adenoviruses (CG0070), recombinant adenovirus interferon alpha $2 \mathrm{~b}$ with Syn 3 (Instiladrin ${ }^{\circledR}$ [nadofaragene firadenovec], FKD Therapies Oy, Kuopio, Finland), modified herpes simplex virus (talimogene laherparepvec [TVEC]), and fusion proteins (Vicinium ${ }^{\circledR}$, Sesen Bio, Inc., Cambridge, MA) are some examples. Additionally, as a result of KEYNOTE-057, the FDA recently approved the first systemic therapy, pembrolizumab, for the treatment of patients with BCG unresponsive CIS with or without papillary tumors who are ineligible for or have elected not to undergo cystectomy.

\section{Chemoablation of Bladder Tumor}

The Chemoablation is a potentially attractive alternative to TURBT that may reduce treatment time, cost, and morbidity. Alternatively, others have also advocated office-based fulguration of small, recurrent low grade tumors when feasible and tolerable as part of a risk-adapted strategy of treatment. ${ }^{116-118}$

Upfront (neoadjuvant) chemo ablative treatments like chemo hyperthermia (hyper thermic intravesical chemotherapy (HIVECT), Combat Medical Ltd., UK) and reverse thermal gel formulated with MMC (UGN102, UroGen Pharma, Inc., Princeton, NJ) are increasingly being explored as alternatives to repeat office or ambulatory procedures in patients with established, recurrent low-grade tumors.

Upfront (neoadjuvant) chemo ablative treatments like chemo hyperthermia and reverse thermal gel formulated with MMC are increasingly being explored as alternatives to repeat office or ambulatory procedures in patients with established, recurrent lowgrade tumors. Active surveillance has also been suggested as a safe and cost-effective alternative for highly selected patients. ${ }^{118}$

HIVEC (Hyper thermic Intra Vesical Chemotherapy) And UGN-102 (Mitomycin sustained release)

\section{Early Data}

HIVEC is neoadjuvant intravesical instillation of heated MMC delivered in a recirculated fashion by a proprietary system called the Bladder Recirculation System (BRS) from Combat Medical. Initial study of the system consisted of 8 courses (60 min dwell/ recirculation time using $80 \mathrm{mg}$ of MMC diluted in 50 $\mathrm{mL}$ of distilled water at $43 \pm 1^{\circ} \mathrm{C}$ ) in 24 patients with intermediate- or high-risk NMIBC delivered in the neoadjuvant setting with TURBT 2 weeks later. ${ }^{33}$ Of these, 15 patients $(62.5 \%)$ had a complete response and 8 additional patients $(33.3 \%)$ showed a partial response. The 4-year cumulative incidence of recurrence was $20.8 \%$ and the agent was well tolerated with $97 \%$ of patients completing all scheduled instillations.

UGN-102 is a reverse thermal hydrogel that is formulated with MMC (UroGen Pharma). It is currently being studied (NCT03558503) as a chemoablative therapy in lieu of TURBT or surgical tumor destruction in patients with low-grade bladder cancer that meet 1 to 2 of 3 criteria for AUA Intermediate Risk NMIBC: multifocal, lesion $>3 \mathrm{~cm}$, and recurrence within 12 months. Because patients with low-grade intermediate-risk NMIBC are at low risk of progression ( $5 \%$ at 2 y) and high recurrence risk ( $\sim 40 \%$ at $2 y)$, the proposed benefit of this agent is two-fold: drug delivery will be improved by greater surface contact time and the morbidity and cost associated with TURBT will be reduced. ${ }^{119}$

The active study is a multicenter, single-arm Phase IIb that is treating patients with UGN-102 weekly for 6 weeks with the primary endpoint being a complete response at 3 months. A complete response was defined as negative cystoscopy, negative cytology, and negative biopsy if possible visual evidence of disease is noted. Secondary endpoints include 12-month durability and safety. Updated interim analysis of 63 patients ( 38 men, 25 women; age range, 33-96) demonstrated a 3-month complete response (CR) rate of $65 \%$ (41/63). Of those with a CR, 97\% (31/32) patient and 85\% (17/20) remained free of disease at 6 and 9 months, respectively. ${ }^{34}$ Mild or moderate adverse events were common, such as dysuria (38\%) and hematuria $(16 \%) .{ }^{119}$ 


\section{Choosing an Adjuvant Treatment}

Bacillus Calmette-Guerin (BCG )

Bacillus Calmette-Guerin was developed in 1921 as a tuberculosis vaccine; BCG is a live attenuated strain of Mycobacterium bovis. Even though it is understood that BCG exerts its antitumor effect through immune activation, the exact mechanism of action has not been fully elucidated. Successful BCG therapy requires an intact immune system and direct contact with live BCG, which is internalized by urothelial and dendritic cells, releasing a number of cytokines, including interleukin6 (IL-6) and granulocyte-macrophage colonystimulating factor (GM-CSF)10-12]. Multiple metaanalyses have demonstrated the superiority of adjuvant BCG for preventing recurrences compared to TURBT alone or TURBT plus intravesical chemotherapy. ${ }^{8}$

Despite the efficacy of intravesical BCG for NMIBC, up to $80 \%$ of treated patients will suffer a recurrence, and up to $45 \%$ may progress to muscle-invasive disease within 5 years[12]. BCG failure refers to NMIBC that recurs or progresses within 6 months of BCG therapy. ${ }^{13}$ European Association of Urology and the American Urological Association (AUA) guidelines still recommend radical cystectomy for patients for whom BCG has failed. ${ }^{14-15}$

Contemporary trials are primarily focused on novel therapies as second-line agents for BCG failures, with BCG therapy failing in up to $50 \%$ of patients in some series. ${ }^{16}$ Novel therapies as alternative first-line agents have also been considered because of concerns about BCGs efficacy and recent shortages of BCG.

\section{Recombinant BCG (rBCG)}

Genetically engineered recombinant BCG (rBCG) strains have been developed to overcome some of the limitations of conventional BCG therapy. Currently, the two major rBCG strategies are T-helper 1 (Th1) cytokine-based rBCG and BCG-Subcomponent-based rBCG.

A Th1 immunologic response is essential for BCG to produce its antitumor effect. This has led to the development of genetically manipulated BCG strains to secrete Th1 cytokines as a strategy to enhance its effective therapy. These strategies have included IL-2, IL-12, IL-18, interferon (IFN-á), and IFN-ã secreting rBCG. ${ }^{17}$ Studies have shown these strains can be effective in increasing IFN-ã production, improving antigen-specific proliferation, eliciting higher levels of
Th1 cytokines, and enhancing antitumor activity. ${ }^{18-}$ ${ }^{22}$ Th1 cytokine-based rBCG strains may have the potential of enhancing BCG for treatment of NMIBC; further clinical trials in humans are necessary.

Using nonlive immunologically active BCG subcomponents, including BCG cell wall and various BCG proteins and antigens, is currently being researched as a method for inducing the same immune response as live BCG in the treatment of NMIBC while possibly avoiding live BCG-associated side effects. Compared with the other subcomponents of BCG, the BCG cell wall has been found to be the most potent Th1 response inducer. ${ }^{23}$ A 2009 multicenter study showed that intravesically administered mycobacterial cell wall-DNA complex (MCC) may be a safe and effective form of treatment in patients with BCGrefractory CIS of the bladder. ${ }^{24}$ A recently completed Phase II/III clinical trial assessing MCC in the treatment of BCG-refractory patients with NMIBC reported significant activity of MCC in these patients with few adverse events. Unfortunately, a Phase III trial evaluating $\mathrm{MCC}$ versus MMC for the treatment of BCG-refractory NMIBC closed early due to poor accrual.

\section{Monoclonal antibodies}

Monoclonal antibodies are a novel immunotherapy strategy targeting tumor-associated antigens (TAAs). CDX-1307 is a monoclonal antibody that targets â-hCG, a TAA elevated in the serum and urine of around 30\%$40 \%$ of cancer patients which may be associated with advanced disease [25]. CDX-1307 consists of B11, a monoclonal antibody against the mannose receptor of antigen-presenting cells (APCs), fused to â-hCG. CDX1307 may be of therapeutic benefit in patients with âhCG expressing bladder cancer through the induction of cellular and humoral responses through antigen presentation of â-hCG on APCs to CD4+ and CD8+ T cells. ${ }^{26}$

Immune checkpoints refer to co-inhibitory receptors expressed in the tumor microenvironment that attenuate tumor-specific effector cells such as T-cells. In addition to immune checkpoints, other mechanisms through which tumors maintain immune tolerance include maintaining protective cell types and producing soluble molecular factors in the tumor microenvironment. Strategies to overcome immune checkpoints represent a novel approach to overcome cancer-induced immune dysfunction. ${ }^{27}$ 
The T-cell activation and proliferation require two major stimulatory signals. The first signal involves the recognition of antigen presented on major histocompatibility complex through APCs by the Tcell receptor (TCR). The second signal is provided through interaction between B7 proteins on APCs and CD28 on the T-cell. Cytotoxic T-lymphocyte-associated antigen 4 (CTLA-4) is expressed by activated T-cells under normal circumstances to prevent excessive Tcell proliferation by competing with the CD28 costimulatory receptor for the secondary $\mathrm{B} 7$ activation signal provided by the APC. CTLA-4 can also be constitutively expressed within the tumor microenvironment to attenuate tumor-specific T-cell responses. ${ }^{28}$ A Phase 3 study showed increased survival in patients with metastatic melanoma receiving ipilimumab, a monoclonal antibody against CTLA-4. A study by Liakou et al. ${ }^{29}$ showed expression of inducible costimulatory (ICOS), a T-cell specific surface molecule structurally similar to CD28, on peripheral blood CD4+ cells in six pre-cystectomy patients with organ-confined bladder cancer treated with ipilimumab. In vitro analysis of these cells showed increased production of IFN-ã as well as an increased effector T-cell to regulatory T-cell ratio. ${ }^{29}$

A Phase 1 trial in 12 presurgical patients with localized urothelial cancer showed that ipilimumab was tolerable in 11 patients, with Grade 1 and 2 toxicities such as rash and diarrhea being the most common adverse effects[30]. CTLA-4 inhibitors do have considerable potential toxicities and have not been evaluated yet in NMIBC but have demonstrated activity in metastatic urothelial carcinoma. ${ }^{31}$

Another immune checkpoint being explored for checkpoint inhibition immunotherapy is MPDL3280A, an anti-programmed death-ligand 1 (PD-L1) monoclonal antibody that inhibits the interaction between PD-L1 and its receptor, PD-1 on T-cells[32]. Expression of PD-L1 acts to maintain immune tolerance and prevent autoimmunity by interacting with PD-1 and inhibiting TCR activation. ${ }^{33}$ Solid tumors, including urothelial tumors, have been shown to exploit this pathway by expressing PD-L1 to promote immune tolerance. ${ }^{34-35}$ PD-L1 acts as a marker for tumor progression and is associated with lower survival rates and its expression on bladder cancer cells was associated with high-grade tumors and was found to be extensively expressed in granulomas of recurrent tumors. ${ }^{36-37}$
In fact, PD-1/PD-L1 expression is found to increase in tumors as they progress from low grade to high grade [36]. MPDL3280A has shown remarkable clinical activity against metastatic urothelial bladder cancer and was not associated with any Grade 4 or 5 adverse events in a recent Phase 1 clinical trial. ${ }^{32,37}$ Other monoclonal antibodies targeting the interaction between PD-1 and PD-L1 include pembrolizumab and atezolizumab and are being evaluated in several ongoing trials. ${ }^{38}$ These trials are evaluating the PD-1/ PD-L1 inhibitors in BCG-unresponsive disease either as single agents or in combination with BCG. One new novel trial being performed at the National Cancer Institute (NCI) will treat patients with combination immunotherapy consisting of an intravesical fusion protein (Vicinium) with systemic checkpoint PD-L1 inhibition (Durvalumab). However, to date, no data have been published demonstrating the clinical efficacy of monoclonal antibodies as checkpoint inhibitors in NMIBC. ${ }^{27,38}$

\section{Vaccines for Bladder Cancer}

Vaccines are becoming an attractive area of research as a potential therapeutic option in the treatment of NMIBC. Vaccines induce an activated immune response in the body, allowing for the targeting of oncoproteins and TAAs by the body's natural defenses. Using the body's activated immune response to target NMIBC may decrease the risk of tumor escape when compared to monoclonal antibody therapy, which targets a single receptor and its downstream targets. In addition, vaccines do not require continual treatment for NMIBC.

Targets of vaccine therapies are currently being evaluated and include various oncoproteins (cancer testes antigens [CTAs], e.g. MAGE-A3), TAAs (e.g. carcinoembryonic antigen [CEA] and mucin-1), heat shock protein 90B1 (hsp90B1), upregulated receptors on NMIBC cells (e.g. CVA21), and surface peptide antigens. Recent and ongoing clinical trials are exploring the efficacy of vaccine treatment in the setting of BCG naïve patients (recMAGE-A3 and CAVATAK), BCG unresponsive patients (ALT-801 and PANVAC), or both (HS-410). ${ }^{38}$

MAGE-A3 is a CTA expressed in up to $40 \%$ of patients with NMIBC, making this an attractive target for therapy. ${ }^{39}$ A Phase I trial combining BCG, recMAGEA3 (a MAGE-A3 vaccine), and adjuvant AS15 (immunostimulant) recently demonstrated "safety and tolerability" in high-grade Ta, T1, or Tis patients and an increase of vaccine-specific T-cells in the bladder. ${ }^{40}$ 
Of note, the vaccine-specific T-cells were only present in the urine of patients who received combination therapy with intravesical BCG. This suggests that combination therapy requires an intravesical treatment to see the effect of vaccination, vaccine-specific T cells, in the urine..

Percutaneous BCG was originally developed as a vaccine against Mycobacterium tuberculosis infection. ${ }^{41}$ Its anticancer effect has been known since 1959, but it was not until 1976 that BCG was reported to have successfully treated bladder cancer

Nearly $40 \%$ of patients treated with BCG will develop positive tuberculin skin tests. In addition, it is documented that patients with a preexisting positive tuberculin skin test have better recurrence-free survival (RFS) rates than those without a positive test after intravesical BCG reported therapy. priming patients with percutaneous BCG seemed to attenuate the immune response[11]. This prompted a current Phase II trial to evaluate the efficacy of percutaneous BCG and intravesical BCG by measuring the complete response rate of therapy at 12 months. The results are pending.

Coxsackievirus A21 (CVA21) (CAVATAK) targets NMIBC by binding CVA21 receptors (intracellular adhesion molecule-1) that are up regulated on NMIBC cells and promote rapid cell lysis through decayaccelerating factor. ${ }^{42}$

CAVATAK was further noted to up regulate I-CAM receptors creating a stronger treatment response when combined with intravesical MMC in a recent study. This Phase II trial reported that treatments were well tolerated and a specific antitumor immune response was noted in 16 patients' pre transurethral resection of the bladder tumors. ${ }^{43}$

ALT-801 is a fusion protein (IL-2 and TCR) that recognizes surface antigens derived from p53 in vivo in p53+/HLA-A2+ tumor xenografts. ${ }^{43}$ A current Phase $\mathrm{Ib} / \mathrm{II}$ trial is investigating safety, tolerability, and cancer recurrence rates up to 13 weeks in patients with highgrade Ta, T1, or Tis NMIBC treated with ALT-801 and IV gemcitabine.

PANVAC is a pox viral vector-based vaccine that contains the TAAs CEA and mucin- 1 as well as three T-cell co-stimulatory molecules. There is a current ongoing Phase II trial studying the 12-month RFS of two treatment arms in BCG-refractory patients: the combination of PANVAC and BCG and BCG only.
Preliminary immune analysis data for the first 16 patients were reported at the AUA annual meeting in 2017.

In the PANVAC and BCG combination arm, an increase in CD8 T-cells, antigen-specific T-cell responses to CEA and MUC-1, and an increase in an antigen-specific Tcell response to brachyury were reported. The T-cell response to brachyury is of particular importance as an immune response against brachyury may potentially trigger an antitumor response cascade as brachyury is a transcription factor involved in epithelial to mesenchymal transition. There was no difference in CD4 cell counts or RFS between the two arms. ${ }^{4}$

HS-410 (Vesigenurtacel-L) is a vaccine containing multiple TAAs (e.g. hsp90B1 and gp96). GP96 antigen complexes are known to be efficient stimulators of CD8+ cytotoxic T-cells. HS-410 and BCG may have synergistic effects in the treatment of NMIBC. The Phase II clinical trials were recently reported at a major meeting evaluating the primary endpoint of 1-year RFS across three study arms. Preliminary results of the 78 patients enrolled reported that RFS rates for BCG and low-dose HS-410, BCG, and high-dose HS-410, and BCG and placebo were $65.4 \%, 65.45 \%$, and $76.9 \%$, respectively. The investigators concluded that HS- 410 was well tolerated and no adverse events related to the vaccine were reported. There was no statistically significant difference in RFS between HS-410 and placebo; however, only the patients exposed to vaccine developed immune responses to tumor-associated peptides. ${ }^{45}$

Although no clinical difference was seen in the three vaccine trials highlighted (MAGE-A3, PANVAC, and HS-410) in patients with or without BCG, all demonstrated that vaccines in combination with BCG led to an "increased immune response." As additional study results are presented, we will be able to better understand the context, in which vaccines may play a role in the treatment of NMIBC. It may be safe to conclude that vaccines may have little efficacy or immunological enhancement as monotherapy.

\section{Cytotoxic chemotherapy}

Epirubicin is a doxorubicin derivative commonly used as a chemotherapy agent in Europe and Japan postTURBT, especially in patients with low and intermediate-risk NMIBC. ${ }^{[38]}$ However, despite an increased risk of toxicity, BCG immunotherapy has 
been shown to be superior to epirubicin in prolonging time to the first recurrence, preventing distant metastasis, and improving overall survival in patients with intermediate- and high-risk NMIBC. ${ }^{46}$

A Phase IV trial is currently recruiting participants to evaluate the efficacy of immediate intravesical instillation of epirubicin after TURBT in patients with intermediate and high-risk NMIBC by measuring the rates of recurrence, progression, and/or death from cancer within the $1^{\text {st }}$ year after complete TURBT.

The antitumor agent MMC, which exerts its effects through DNA alkylation, has been found to improve recurrence rates $(42 \%$ vs. $58 \%$ ) when combined with BCG therapy in a Phase II trial. ${ }^{47}$

In an ongoing Phase III trial, the efficacy of MMC and BCG combination intravesical therapy in high-risk patients with NMIBC is being evaluated by assessing the disease-free survival over 5 years. A recently completed early Phase I clinical trial found that external deep pelvic hyperthermia is a safe and effective method of heating the bladder in patients undergoing intravesical MMC. In this study, 15 patients with BCG-refractory NMIBC underwent external deep pelvic hyperthermia combined with intravesical MMC. The reported adverse events were all minor (Grade 2 or less), with no systemic toxicity observed. ${ }^{48}$

Even though multi agent therapy is regularly used systemically in patients with metastatic disease, there is growing interest in using multiple agents intravesically, and recent studies have shown encouraging outcomes.

For example, disease-free rates of $54 \%$ at 1 year and $34 \%$ at 2 years have been demonstrated in one study of the utility of the intravesical combination of gemcitabine with docetaxel in 45 patients with NMIBC recurrence after BCG therapy and disease-free rates of $50 \%$ at 1 year have been demonstrated in a multiinstitutional retrospective study of high-risk patients with NMIBC treated with sequential intravesical gemcitabine followed by MMC. ${ }^{49-50}$

A Phase I trial is currently underway to evaluate the safety of an intravesically administered a multidrug regimen of cabazitaxel, gemcitabine, and cisplatin (CGC) in the treatment of BCG-resistant NMIBC. Early results show that intravesical CGC appears to be well tolerated in patients with NMIBC, with five patients experiencing at least one Grade 1 toxicity and the remaining four patients experiencing at least one Grade 2 toxicity. ${ }^{51}$

\section{Targeted Therapy}

Oportuzumab monatox $(\mathrm{OM})$ is a recombinant fusion protein of humanized antiepithelial cell adhesion molecule antibody linked to Pseudomonas exotoxin. In high-grade tumors, including bladder cancer, the epithelial cell adhesion molecule surface antigen is usually overexpressed, allowing OM to specifically target tumor cells. ${ }^{42}$

Two OM dosing strategies were evaluated in a Phase II trial in BCG-refractory CIS patients and found complete response rates of $26.7 \%$ and $15.6 \%$ at 6 - and 12-month intervals, respectively.

This has prompted a new Phase I trial that will open at the NCI this fall for BCG unresponsive HG NMIBC that will treat patients with intravesical OM combined with systemic durvalumab. The trial will evaluate safety, but secondary endpoints will look at efficacy and immunologic correlates. ${ }^{52}$

Other examples of targeted therapy include BGJ398, sunitinib, enzalutamide, ethacrynic acid, and tamoxifen. BGJ398 is a tyrosine kinase inhibitor targeting FGFR3 and found to be activated in approximately $75 \%$ of all cases of NMIBC. ${ }^{53}$

A Phase I/II trial to evaluate the safety and efficacy of oral administration of BGJ398 in BCG-refractory patients with NMIBC is currently underway. Sunitinib functions by inhibiting various key tyrosine kinases including vascular endothelial growth factor receptors 1,2 , and $3 .^{54}$

The combination of oral sunitinib and intravesical BCG is being evaluated in high-risk patients with NMIBC in an ongoing Phase II trial. The most recent data from the study show that $72 \%$ of patients have a complete response at 3 months with $77 \%$ RFS and 100\% progression-free survival at 24 months. ${ }^{55}$

Enzalutamide, an androgen receptor (AR) antagonist that has been used in the treatment of metastatic castrate-resistant prostate cancer, has been shown to inhibit bladder cancer proliferation, migration, and invasion in AR+ cell lines. ${ }^{56}$

The efficacy of enzalutamide in preventing bladder cancer recurrence in patients with both $\mathrm{AR}+$ or AR ${ }^{\mathrm{TM}}$ NMIBC will be evaluated by looking at recurrence rate over a 12-month period in an ongoing Phase 2 study. Ethacrynic acid is recognizable as a commonly used loop diuretic. However, it has also been shown to be cytotoxic through inhibition of glutathione S-transferase and Wnt/â-catenin signaling, 
and dysregulation of which has been implicated in various tumors including bladder cancer. ${ }^{57-58}$

The recently completed Phase I trial aims to evaluate the safety and efficacy of ethacrynic acid when given immediately before TURBT in patients with NMIBC. Tamoxifen is a nonsteroidal selective estrogen-receptor modulator mainly used in the treatment of breast cancer. However, a preclinical study has shown that it is also effective in reducing the incidence of bladder cancer in mice. ${ }^{59}$

An ongoing Phase II trial is evaluating the efficacy of oral tamoxifen citrate in patients with low-tointermediate-risk NMIBC by assessing for the clinical response of the marker lesion over a 4-year period.

\section{Immunotherapy}

Imiquimod (an imidazoquinoline) is an existing topical treatment for basal cell carcinoma, actinic keratosis, and condylomas. It has been modified for intravesical use and is being investigated in the treatment of NMIBC as TLR7 is also found in bladder cancer. ${ }^{60-61}$

A 2013 single-center Phase I trial found TMX-101 to be safe with low systemic effects and mild side effects typically limited to the genitourinary tract. ${ }^{62}$

A 2017 prospective nonrandomized, multicenter, Phase II trial demonstrated continued safely and an immune response as evidenced by increased levels of urinary cytokines (IL-6 and IL-18) in 12 Ta to T1 patients. In addition, 2 out of 12 patients reported negative bladder biopsies at 6-week follow-up post treatment. ${ }^{63}$

ALT-803 is a recombinant immunomodulating fusion (IL-15 analog + IL15-RaFc) protein that is 25 times more active than IL-15 in vivo and a demonstrated ability to activate natural killer and CD8+ T-cells through local cytokines. ${ }^{64}$

In a rodent NMIBC model, ALT-803+ BCG was shown to reduce tumor burden (by $46 \%$ ), produce a local cytokine response (IL-1á, IL-1 â, and RANTES), activate NK cells, and reduce angiogenesis (by 76\%) when compared to the control group. ${ }^{65}$

ALT-803+ BCG was shown to be more effective than either BCG or ALT-803 alone. A Phase I and II study is currently underway. The group undertaking the clinical trials released a case report ahead of their published Phase I and II results. In it, a 92 man with a history of Ta high-grade NMIBC and recurrent disease post-BCG therapy remains cancer free 19 months after treatment with ALT-803+ BCG. ${ }^{65}$
Lenalidomide, an immunomodulator, is a thalidomide derivative with a known tumoricidal activity that has shown improved time to progression and increases in overall survival in relapsing multiple myeloma Phase III trials. ${ }^{66-67}$

These properties make it an attractive potential treatment option for NMIBC, for which it has undergone a Phase I murine-based clinical trial. They reported that lenalidomide + BCG produced significant antitumor effects: increasing cell death, decreasing tumor volume, and reducing angiogenesis in tumors. 66-67

Atezolizumab is a humanized monoclonal antibody capable of selectively antagonistically binding programmed death ligand-1 (PD-L1) on tumor cells. PD-L1 decreases T-cell activity and is broadly expressed across a wide array of malignancies, providing survival benefits for tumors overexpressing PD-L1. ${ }^{68-69}$

One multicenter, single-arm, Phase II study demonstrated that atezolizumab was well tolerated and provided antitumor responses in 310 patients with locally advanced or metastatic urothelial carcinoma who previously failed platinum chemotherapy. In addition, patients' tumor PD-L1 levels were measured which showed that the higher the expression of PDL1, the more likely the patient responded to treatment. ${ }^{68-69}$

Pembrolizumab (MK-3475) is a humanized monoclonal antibody that binds the PD-L1 receptor but this time on the immune $(\mathrm{T})$ cells - preventing them from interacting with their ligands ${ }^{70}$. In a Phase $\mathrm{Ib}$ clinical trial, pembrolizumab was reported to be safe and tolerated well in 33 patients. ${ }^{71}$

In a 2017 randomized Phase III trial, pembrolizumab showed significantly longer overall survival than chemotherapy (10.3 pembrolizumab and 7.4 chemotherapy) with less adverse events from treatment in 542 patients with recurrent advanced urothelial cancer postplatinum chemotherapy treatment. $^{72}$

As a result, pembrolizumab was recently approved by the Food and Drug Administration as a second-line treatment for metastatic urothelial carcinoma. ${ }^{73}$

Several PD-1/PD-L1 inhibitors such as durvalumab, atezolizumab, and pembro lizumab are being evaluated in clinical trials for non-muscle invasive 
disease given their activity in metastatic urothelial cancer.

\section{Gene Therapy}

CG0070 is a recombinant adenovirus that allows for selective viral replication in tumor cells and local production of GM-CSF by targeting the often deregulated retinoblastoma tumor suppressor pathway. ${ }^{74}$

The intravesical instillation of CG-0700 has been shown to be safe, with a complete response rate of $48.6 \%$ at 10.4 months. ${ }^{74}$ An ongoing Phase III trial is looking at complete response lasting at least 12 months to evaluate the efficacy of CG-0070 in patients with NMIBC who have failed BCG therapy.

rAd-IFN/Syn-3 (Instiladrin) is a nonreplicating recombinant adenovirus vector containing the human IFN alpha-2b (IFNá2b) gene and the addition of Syn-3 significantly augments adenoviral-mediated transduction of normal urothelium and NMIBC, resulting in high, durable urine IFNá concentrations and tumor regression. ${ }^{75}$

The Phase I and II trials involving rAd-IFN/Syn-3 have demonstrated detectable levels of IFN-á in urine, as well as RFS, was $35 \%$ at 12 months in the Phase II trial. ${ }^{75-76}$ The current Phase III trial evaluates the efficacy of rAD-IFN/Syn-3 in BCG-unresponsive patients with NMIBC by looking at the event-free survival at 12 months.

VPM1002BC is a live genetically modified $M$. bovis BCG that expresses the bacterial toxin listeriolysin. This recombinant form of BCG has demonstrated higher levels of the antigen-specific memory T-cell and $\mathrm{T}$ follicular helper $\mathrm{T}$ cells associated with specific antibody responses. This is thought to be due to improved antigen presentation as a result of the pore-forming ability of the toxin. ${ }^{77}$

An ongoing Phase I/II trial is evaluating the safety, tolerability, and efficacy of intravesical installation of VMP1002BC in patients with recurrent NMIBC.

\section{Chemo Immuno radiation}

Trimodality therapy (TMT) refers to TURBT followed by chemoradiation involving agents such as 5-FU, MMC, and cisplatin. Extensive studies have shown similar overall survival with TMT as radical cystectomy in patients with muscle-invasive bladder cancer. $^{78}$
Recent studies have shown that TMT may also be effective in the recurrent high-grade T1 tumor, with one study reporting an $88 \%$ complete response rate and a $19 \%$ progression rate in patients with T1G3 NMIBC tumors. ${ }^{79}$

An ongoing Phase II trial (RTOG 0926) aims to evaluate the efficacy of TMT in patients with NMIBC with persistent $\mathrm{T} 1$ disease who have failed BCG therapy by evaluating the rate of freedom from radical cystectomy at 3 years.

Radiation therapy (RT) amplifies tumor immunogenicity and may be capable of inducing or potentiating a systemic antitumor immune response. Immuno radiotherapy (IRT) may also induce immuneinhibiting responses in the microenvironment (increase of PD-L1, TGF-â, or inhibitory immune cells such as regulatory T-cells, alternatively activated macrophages, and myeloid-derived suppression cells [MDSCs]) that act against the effective antitumor response. ${ }^{80}$

Preclinical synergistic local (radiosensitizing immunotherapy) and distant antitumor activity were shown with the combination of irradiation and antiPD-L1. This effect was associated with a decrease in local accumulation of tumor-infiltrating MDSC that inhibits of T-cell function. ${ }^{80}$

One of the first clinical results assessing an immune checkpoint blocker with concomitant RT was recently completed and found that concurrent palliative RT with durvalumab was well tolerated in ten patients with histologically or cytologically confirmed inoperable or metastatic cancers. ${ }^{81}$

A combined Phase Ib/II study of concurrent durvalumab and RT followed by adjuvant durvalumab in patients with urothelial cancer (T2-4, N0-2, MO) of the bladder is currently underway with primary outcome measures of safety assessment, progressionfree survival after 1 year, and disease control rate after 15 months.

There are numerous uncertainties associated with IRT. The timing and the radiation dose per fraction seem to be important parameters determining the modulation of the immune response. Fractionated RT could be more appropriate than single dose RT. There is also an unmet need of surrogate markers to predict or assess the immune response. PD-L1 "positive" tumors have shown trends toward increased rates of response to PD-L1 blockade. ${ }^{6}$ 


\section{Intravesical Drug Delivery Systems}

Nanoparticle albumin-bound paclitaxel (nabPaclitaxel) was investigated in a Phase II trial using patients with recurrent NMIBC (Tis, Ta, and T1) after BCG therapy and a Phase III trial currently underway. Paclitaxel stabilizes microtubules, arresting them at $\mathrm{G}_{2}{ }^{-}$ $\mathrm{M}$ in the cell cycle. After adding albumin (creating nabpaclitaxel), the nanoparticle's solubility is increased fivefold and can use albumin receptor-mediated transport for increased delivery to tumor cells. ${ }^{38,82}$

Long-term follow-up data from the Phase II trial (medium 41-month follow-up) on 28 patients showed cancer-specific survival rate of $91 \%$ (18\% disease free) at 5 years. ${ }^{82}$

Some drug targets appear promising but their short half-lives limit exposure to the urothelium and render them ineffective. A few trials explore c-administering treatment medication with other compounds that target the mucous membrane of the bladder to add synergistic effects. A novel trial used Syn 3 (enhancer: binding and stabilization) to aid in rAd-IFN (recombinant adenovirus producing interferon alpha$2 b$ ) intravesical delivery in refractory NMIBC patients. Commercially known as Instiladrin (rAd-IFN with Syn3), Phase II results showed that rAd-IFN with Syn3 was tolerated well with no Grade 4 or 5 adverse events, and $35 \%$ (14 patients) were free of high-grade recurrence at 12 months (AA). ${ }^{83}$

Other mucoadhesive nanogels are beginning to be investigated in the delivery of NMIBC delivery of therapy. Hydrophobic drugs are known to have greater penetration of the urothelium than hydrophilic and thus creating hydrophobic carriers of drugs may produce better treatment result ${ }^{84}$

Mucoadhesive nanogels showed promise in animal models, reportedly aiding uptake of docetaxel through endocytosis and releasing $75 \%$ of loaded drug over 9 days. ${ }^{85}$

\section{Chemohyperthermia}

Combining NMIBC treatment with external heat before administration has shown that heat + mitomycin- $\mathrm{C}$ is more efficacious than either treatment alone. ${ }^{[86]}$ Combined chemohyperthermia (CHT) for the treatment of NMBIC as an invasive procedure was first reported safe and reproducible in 2014, with hyperthermia doses of $42^{\circ} \mathrm{C} \pm 2^{\circ} \mathrm{C}$ for $40-60 \mathrm{~min}$ through BSD-2000 hyperthermia system (eternal radiofrequency) ${ }^{48,87}$.
A randomized controlled trial comparing $\mathrm{CHT}+$ MMC against BCG as an adjuvant therapy in intermediate- and high-risk NMIBC showed that CHT is safe and effective with a significantly higher RFS rate at 24 months. ${ }^{88}$

\section{Active surveillance for NMIBC( nonmuscle invasive bladder cancer)}

Nonmuscle invasive bladder cancer (NMIBC) is known to be a heterogeneous malignancy that requires varying treatment modalities and follow-up schedules. Lowgrade Ta papillary tumors are categorized as low-risk NMIBC because of their favorable prognosis. There is an expanding movement that over diagnosis and overtreatment should be avoided considering the economic impact and the patients' quality of life.

Urologists are familiar with the concept of AS for urogenital malignancies such as low-risk prostate cancer ${ }^{126-127}$ and renal cell masses. ${ }^{126-128}$ Although the initial assessment of AS for bladder cancer was reported more than 10 years ago. ${ }^{129} 129$ it is still an uncommon option, but it may be ideal in selected patients.

It has been over 10 years since the initial assessment of active surveillance for low-risk NMIBC suggested its feasibility and safety. However, urologists are still unfamiliar with this treatment option, which can be ideal in appropriately selected patients. In this review article, we focus on active surveillance for low-risk NMIBC and discuss the evidence and rationale for this treatment option. There are several issues to resolve in order to advocate active surveillance as a standard option in selected patients. A specific follow-up protocol including intervals of cystoscopy, urine cytology, urine markers, and other radiographic examinations need to be optimized and validated. Large-scale randomized clinical trials are required to prove the real benefit of AS for well-selected patients. Similarly, we need to make maximal efforts to obtain significantly reliable biomarkers for AS in the urine or sera and to establish monitoring systems. ${ }^{129}$

\section{Conclusion}

NMIBC remains a very challenging disease to treat not only because of the high rates of recurrence and progression associated with current therapies but also due to the extensive follow-up necessary after diagnosis and initial treatment. As discussed in this review, however, many novel therapies for NMIBC are currently being developed and tested in clinical trials 
ranging from vaccines to immunomodulators and targeted therapies. The ongoing efforts to develop new therapeutic approaches for NMIBC look very promising and may help develop a new paradigm of NMIBC treatment in the near future.

Conflicts of interest: There are no conflicts of interest.

\section{References}

1. Ploeg M, Aben KK, Kiemeney LA. The present and future burden of urinary bladder cancer in the world. World J Urol 2009;27:28993. [PUBMED]

2. Siegel RL, Miller KD, Jemal A. Cancer statistics, 2017. CA Cancer J Clin 2017;67:7-30. [PUBMED]

3. Kaufman DS, Shipley WU, Feldman AS. Bladder cancer. Lancet 2009;374:239-49. [PUBMED]

4. Veeratterapillay R, Heer R, Johnson MI, Persad $\mathrm{R}$, Bach C. High-risk non-muscle-invasive bladder cancer-therapy options during intravesical BCG Shortage. Curr Urol Rep 2016;17:68. [PUBMED]

5. Botteman MF, Pashos CL, Redaelli A, Laskin B, Hauser R. The health economics of bladder cancer: A comprehensive review of the published literature. Pharmacoeconomics 2003;21:131530. [PUBMED]

6. Hall MC, Chang SS, Dalbagni G, Pruthi RS, Seigne JD, Skinner EC, et al. Guideline for the management of nonmuscle invasive bladder cancer (stages Ta, T1, and Tis): 2007 update. J Urol 2007;178:2314-30. [PUBMED]

7. Sylvester RJ. Bacillus calmette-guérin treatment of non-muscle invasive bladder cancer. Int J Urol 2011;18:113-20. [PUBMED]

8. Packiam VT, Johnson SC, Steinberg GD. Nonmuscle-invasive bladder cancer: Intravesical treatments beyond Bacille Calmette-Guérin. Cancer 2017;123:390-400. [PUBMED]

9. Stein JP, Lieskovsky G, Cote R, Groshen S, Feng AC, Boyd S, et al. Radical cystectomy in the treatment of invasive bladder cancer: Long-term results in 1,054 patients. J Clin Oncol 2001;19:66675. [PUBMED]

10. Zbar B, Tanaka T. Immunotherapy of cancer: Regression of tumors after intralesional injection of living Mycobacterium bovis. Science 1971;172:271-3. [PUBMED]
11. Redelman-SidiG, Glickman MS, Bochner BH. The mechanism of action of BCG therapy for bladder cancer - a current perspective. Nat Rev Urol 2014;11:153-62. [PUBMED]

12. van Rhijn BW, Burger M, Lotan $Y$, Solsona E, Stief CG, Sylvester RJ, et al. Recurrence and progression of disease in non-muscle-invasive bladder cancer: From epidemiology to treatment strategy. Eur Urol 2009;56:430-42. [PUBMED]

13. Yates DR, Brausi MA, Catto JW, Dalbagni G, Rouprêt M, Shariat SF, et al. Treatment options available for bacillus Calmette-Guérin failure in non-muscle-invasive bladder cancer. Eur Urol 2012;62:1088-96.

14. Babjuk M, Böhle A, Burger M, Capoun O, Cohen $\mathrm{D}$, Compérat EM, et al. EAU guidelines on nonmuscle-invasive urothelial carcinoma of the bladder: Update 2016. Eur Urol 2017;71:447-61.

15. Chang SS, Boorjian SA, Chou R, Clark PE, Daneshmand S, Konety BR, et al. Diagnosis and treatment of non-muscle invasive bladder cancer: AUA/SUO guideline. J Urol 2016;196:10219. [PUBMED]

16. Brake M, Loertzer H, Horsch R, Keller H. Longterm results of intravesical bacillus CalmetteGuérin therapy for stage T1 superficial bladder cancer. Urology 2000;55:673-8. [PUBMED]

17. Muthigi A, George AK, Brancato SJ, Agarwal PK. Novel immunotherapeutic approaches to the treatment of urothelial carcinoma. Ther Adv Urol 2016;8:203-14. [PUBMED]

18. Slobbe L, Lockhart E, O'Donnell MA, MacKintosh C, De Lisle G, Buchan G, et al. An in vivo comparison of bacillus Calmette-Guérin (BCG) and cytokine-secreting BCG vaccines. Immunology 1999;96:517-23.

19. Young S, O'Donnell M, Lockhart E, Buddle B, Slobbe L, Luo Y, et al. Manipulation of immune responses to Mycobacterium bovis by vaccination with IL-2- and IL-18-secreting recombinant bacillus Calmette Guerin. Immunol Cell Biol 2002;80:209-15.

20. Brunda MJ, Luistro L, Warrier RR, Wright RB, Hubbard BR, Murphy M, et al. Antitumor and antimetastatic activity of interleukin 12 against murine tumors. J Exp Med 1993;178:122330. [PUBMED] 
21. Nastala CL, Edington HD, McKinney TG, Tahara $\mathrm{H}$, Nalesnik MA, Brunda MJ, et al. Recombinant IL-12 administration induces tumor regression in association with IFN-gamma production. J Immunol 1994;153:1697-706. [PUBMED]

22. Murray PJ, Aldovini A, Young RA. Manipulation and potentiation of antimycobacterial immunity using recombinant bacille Calmette-Guérin strains that secrete cytokines. Proc Natl Acad Sci U S A 1996;93:934-9. [PUBMED]

23. Zlotta AR, Van Vooren JP, Denis O, Drowart A, Daffé $\mathrm{M}$, Lefèvre $\mathrm{P}$, et al. What are the immunologically active components of bacille Calmette-Guérin in therapy of superficial bladder cancer? Int J Cancer 2000;87:844-52.

24. Morales A, Phadke K, Steinhoff G. Intravesical mycobacterial cell wall-DNA complex in the treatment of carcinoma in situ of the bladder after standard intravesical therapy has failed. J Urol 2009;181:1040-5. [PUBMED]

25. Iles RK. Ectopic hCGbeta expression by epithelial cancer: Malignant behaviour, metastasis and inhibition of tumor cell apoptosis. Mol Cell Endocrinol 2007;260-262:264-70. [PUBMED]

26. Morse MA, Bradley DA, Keler T, Laliberte RJ, Green JA, Davis TA, et al. CDX-1307: A novel vaccine under study as treatment for muscleinvasive bladder cancer. Expert Rev Vaccines 2011;10:733-42. [PUBMED]

27. Maruf M, Brancato SJ, Agarwal PK. Nonmuscle invasive bladder cancer: A primer on immunotherapy. Cancer Biol Med 2016;13:194205. [PUBMED]

28. Rothschild SI, Thommen DS, Moersig W, Müller P, Zippelius A. Cancer immunology Development of novel anti-cancer therapies. Swiss Med Wkly 2015;145:w14066.

29. Liakou CI, Kamat A, Tang DN, Chen H, Sun J, Troncoso P, et al. CTLA-4 blockade increases IFNgamma-producing CD4+ICOShi cells to shift the ratio of effector to regulatory $\mathrm{T}$ cells in cancer patients. Proc Natl Acad Sci U S A 2008;105:1498792. [PUBMED]

30. Carthon BC, Wolchok JD, Yuan J, Kamat A, Ng Tang DS, Sun J, et al. Preoperative CTLA-4 blockade: Tolerability and immune monitoring in the setting of a presurgical clinical trial. Clin Cancer Res 2010;16:2861-71. [PUBMED]

31. Kurtoglu M, Davarpanah NN, Qin R, Powles T, Rosenberg JE, Apolo AB, et al. Elevating the horizon: Emerging molecular and genomic targets in the treatment of advanced urothelial carcinoma. Clin Genitourin Cancer 2015;13:41020.

32. Powles T, Eder JP, Fine GD, Braiteh FS, Loriot $Y$, Cruz C, et al. MPDL3280A (anti-PD-L1) treatment leads to clinical activity in metastatic bladder cancer. Nature 2014;515:558-62. [PUBMED]

33. Eppihimer MJ, Gunn J, Freeman GJ, Greenfield EA, Chernova T, Erickson J, et al. Expression and regulation of the PD-L1 immunoinhibitory molecule on microvascular endothelial cells. Microcirculation 2002;9:133-45. [PUBMED]

34. Zou W, Chen L. Inhibitory B7-family molecules in the tumour microenvironment. Nat Rev Immunol 2008;8:467-77. [PUBMED]

35. Hafez N, Petrylak DP. Could PD-L1 prove to be an effective therapeutic target for bladder cancer? Immunotherapy 2015;7:1-2. [PUBMED]

36. nman BA, Sebo TJ, Frigola X, Dong H, Bergstralh EJ, Frank I, et al. PD-L1 (B7-H1) expression by urothelial carcinoma of the bladder and BCGinduced granulomata: Associations with localized stage progression. Cancer 2007;109:1499505. [PUBMED]

37. Nakanishi J, Wada Y, Matsumoto K, Azuma M, Kikuchi K, Ueda S, et al. Overexpression of B7H1 (PD-L1) significantly associates with tumor grade and postoperative prognosis in human urothelial cancers. Cancer Immunol Immunother 2007;56:1173-82.

38. Siddiqui MR, Grant C, Sanford T, Agarwal PK. Current clinical trials in non-muscle invasive bladder cancer. Urol Oncol 2017;35:51627. [PUBMED]

39. Derré L, Cesson V, Lucca I, Cerantola Y, Valerio $\mathrm{M}$, Fritschi $\mathrm{U}$, et al. Intravesical bacillus Calmette Guerin combined with a cancer vaccine increases local T-cell responses in non-muscle-invasive bladder cancer patients. Clin Cancer Res 2017;23:717-25.

40. Gérard C, Baudson N, Ory T, Louahed J. Tumor mouse model confirms MAGE-A3 cancer 
immunotherapeutic as an efficient inducer of long-lasting anti-tumoral responses. PLoS One 2014;9:e94883.

41. Zwerling A, Behr MA, Verma A, Brewer TF, Menzies D, Pai M, et al. The BCG world atlas: A database of global BCG vaccination policies and practices. PLoS Med 2011;8:e1001012.

42. Skelding KA, Barry RD, Shafren DR. Enhanced oncolysis mediated by coxsackievirus A21 in combination with doxorubicin hydrochloride. Invest New Drugs 2012;30:568-81. [PUBMED]

43. Annels NE, Simpson G, Arif M, Denyer M, Iqbal A, Mansfield D, et al. Oncolytic immunotherapy for the treatment of non-muscle invasive bladder cancer using intravesical coxsackievirus A21. J Immunother Cancer 2015;3 Suppl 2:331.

44. Sanford T, Donahue R, Jochems C, Dolan R, Bellfield S, Anderson M, et al. Mp15-10 immunologic response to a therapeutic cancer vaccine (Panvac): Initial results from a randomized Phase 2 clinical trial. J Urol 2017;197:e174.

45. Steinberg GD, Shore N, Lawrence KI, Bailen JL, Bivalacqua TJ, Chamie K. Immune response results of vesigenurtacel-1 (HS-410) in combination with BCG from a randomized phase II trial in patients with non-muscle invasive bladder cancer (NMIBC). J Clin Oncol 2017;35 6 Suppl: 319.

46. Sylvester RJ, Oosterlinck W, van der Meijden AP. A single immediate postoperative instillation of chemotherapy decreases the risk of recurrence in patients with stage Ta T1 bladder cancer: A metaanalysis of published results of randomized clinical trials. J Urol 2004;171:218690. [PUBMED]

47. Hayne D, Stockler M, McCombie SP, Chalasani $\mathrm{V}$, Long A, Martin A, et al. BCG+MMC trial: Adding mitomycin $C$ to BCG as adjuvant intravesical therapy for high-risk, non-muscleinvasive bladder cancer: A randomised phase III trial (ANZUP 1301). BMC Cancer 2015;15:432.

48. Inman BA, Stauffer PR, Craciunescu OA, Maccarini PF, Dewhirst MW, Vujaskovic Z, et al. A pilot clinical trial of intravesical mitomycin-C and external deep pelvic hyperthermia for nonmuscle-invasive bladder cancer. Int J Hyperthermia 2014;30:171-5.
49. Velaer KN, Steinberg RL, Thomas LJ, O'Donnell MA, Nepple KG. Experience with sequential intravesical gemcitabine and docetaxel as salvage therapy for non-muscle invasive bladder cancer. Curr Urol Rep 2016;17:38.

50. Lightfoot AJ, Breyer BN, Rosevear HM, Erickson BA, Konety BR, O'Donnell MA, et al. Multiinstitutional analysis of sequential intravesical gemcitabine and mitomycin $\mathrm{C}$ chemotherapy for non-muscle invasive bladder cancer. Urol Oncol 2014;32:35.e15-9.

51. DeCastro GJ, Wilson S, Pak JS, Abate-Shen CT, Lee SM, Anderson CB. A phase I trial for the use of intravesical cabazitaxel, gemcitabine, and cisplatin (CGC) in the treatment of BCGrefractory nonmuscle invasive urothelial carcinoma of the bladder. J Clin Oncol 2017;35 6 Suppl: 313.

52. Kowalski M, Guindon J, Brazas L, Moore C, Entwistle J, Cizeau J, et al. A phase II study of oportuzumab monatox: An immunotoxin therapy for patients with noninvasive urothelial carcinoma in situ previously treated with bacillus Calmette-Guérin. J Urol 2012;188:17128. [PUBMED]

53. Lindgren D, Liedberg F, Andersson A, Chebil G, Gudjonsson S, Borg A, et al. Molecular characterization of early-stage bladder carcinomas by expression profiles, FGFR3 mutation status, and loss of 9q. Oncogene 2006;25:2685-96. [PUBMED]

54. Gallagher DJ, Milowsky MI, Gerst SR, Ishill N, Riches J, Regazzi A, et al. Phase II study of sunitinib in patients with metastatic urothelial cancer. J Clin Oncol 2010;28:1373-9. [PUBMED]

55. Helfand AM, Lee CT, Hafez K, Hussain M, Liebert M, Daignault S, et al. Phase II clinical trial of intravesical bacillus Calmette-Guerin (BCG) followed by sunitinib for the treatment of highrisk nonmuscle-invasive bladder cancer (NMIBC). J Clin Oncol 2015;33 7 Suppl:293.

56. Kawahara T, Ide H, Kashiwagi E, El-Shishtawy KA, Li Y, Reis LO, et al. Enzalutamide inhibits androgen receptor-positive bladder cancer cell growth. Urol Oncol 2016;34:432.e15-23.

57. Lu D, Liu JX, Endo T, Zhou H, Yao S, Willert K, et al. Ethacrynic acid exhibits selective toxicity to 
chronic lymphocytic leukemia cells by inhibition of the Wnt/beta-catenin pathway. PLoS One 2009;4:e8294. [PUBMED]

58. Majid S, Saini S, Dahiya R. Wnt signaling pathways in urological cancers: Past decades and still growing. Mol Cancer 2012;11:7. [PUBMED]

59. Godoy G, Gakis G, Smith CL, Fahmy O. Effects of androgen and estrogen receptor signaling pathways on bladder cancer initiation and progression. Bladder Cancer 2016;2:12737. [PUBMED]

60. Smith EB, Schwartz M, Kawamoto H, You X, Hwang D, Liu H, et al. Antitumor effects of imidazoquinolines in urothelial cell carcinoma of the bladder. J Urol 2007;177:2347-51. [PUBMED]

61. Arends TJ, Lammers RJ, Falke J, van der Heijden AG, Rustighini I, Pozzi R, et al. Pharmacokinetic, pharmacodynamic, and activity evaluation of TMX-101 in a multicenter phase 1 study in patients with papillary non-muscle-invasive bladder cancer. Clin Genitourin Cancer 2015;13:204-9.e2.

62. Falke J, Lammers RJ, Arentsen HC, Ravic M, Pozzi $\mathrm{R}$, Cornel EB, et al. Results of a phase 1 dose escalation study of intravesical TMX-101 in patients with nonmuscle invasive bladder cancer. J Urol 2013;189:2077-82. [PUBMED]

63. Donin NM, Chamie K, Lenis AT, Pantuck AJ, Reddy M, Kivlin D, et al. A phase 2 study of TMX101, intravesical imiquimod, for the treatment of carcinoma in situ bladder cancer. Urol Oncol 2017;35:39.e1-39.e7.

64. Gomes-Giacoia E, Miyake M, Goodison S, Sriharan A, Zhang G, You L, et al. Intravesical ALT-803 and BCG treatment reduces tumor burden in a carcinogen induced bladder cancer rat model; a role for cytokine production and NK cell expansion. PLoS One 2014;9: e96705. [PUBMED]

65. Huang J, Schisler J, Wong HC, Rosser CJ, Sterbis J. Intravesical ALT-803 for BCG-unresponsive bladder cancer - A case report. Urol Case Rep 2017;14:15-7. [PUBMED]

66. Jinesh GG, Lee EK, Tran J, Kamat AM. Lenalidomide augments the efficacy of bacillus
Calmette-Guerin (BCG) immunotherapy in vivo. Urol Oncol 2013;31:1676-82.

67. Dimopoulos M, Spencer A, Attal M, Prince HM, Harousseau JL, Dmoszynska A, et al. Lenalidomide plus dexamethasone for relapsed or refractory multiple myeloma. N Engl J Med 2007;357:2123-32. [PUBMED]

68. Rosenberg JE, Hoffman-Censits J, Powles T, van der Heijden MS, Balar AV, Necchi A, et al. Atezolizumab in patients with locally advanced and metastatic urothelial carcinoma who have progressed following treatment with platinum-based chemotherapy: A single-arm, multicentre, phase 2 trial. Lancet 2016;387:190920. [PUBMED]

69. Homet Moreno B, Ribas A. Anti-programmed cell death protein-1/ligand-1 therapy in different cancers. Br J Cancer 2015;112:1421-7. [PUB MED]

70. Chen L, Han X. Anti-PD-1/PD-L1 therapy of human cancer: Past, present, and future. J Clin Invest 2015;125:3384-91. [PUBMED]

71. Plimack ER, Bellmunt J, Gupta S, Berger R, Chow LQ, Juco J, et al. Safety and activity of pembrolizumab in patients with locally advanced or metastatic urothelial cancer (KEYNOTE-012): A non-randomised, open-label, phase $1 \mathrm{~b}$ study. Lancet Oncol 2017;18:212-20. [PUBMED]

72. Bellmunt J, de Wit R, Vaughn DJ, Fradet $Y$, Lee JL, Fong L, et al. Pembrolizumab as second-line therapy for advanced urothelial carcinoma. N Engl J Med 2017;376:1015-26. [PUBMED]

73. Three drugs approved for urothelial carcinoma by FDA. Cancer Discov 2017;7:659-60.

74. Boehm BE, Svatek RS. Novel therapeutic approaches for recurrent nonmuscle invasive bladder cancer. Urol Clin North Am 2015;42:15968 , vii. [PUBMED]

75 Dinney CP, Fisher MB, Navai N, O'Donnell MA, Cutler D, Abraham A, et al. Phase I trial of intravesical recombinant adenovirus mediated interferon-á $2 b$ formulated in syn 3 for Bacillus Calmette-Guérin failures in nonmuscle invasive bladder cancer. J Urol 2013;190:850-6.

76 Canter D, Boorjian S, Ogan K, Shore N, Bivalacqua $\mathrm{T}$, Bochner $\mathrm{B}$, et al. Pd17-06 
randomized phase II trial of intravesical adenoviral mediated interferon-á Gene therapy with the excipient syn3 (rad-ifná/syn3) in patients with bcg refractory or relapsing high grade (hg) non muscle invasive bladder cancer (nmibc). J Urol 2015 193:e383.

77. Vogelzang A, Perdomo C, Zedler U, Kuhlmann $S$, Hurwitz R, Gengenbacher M, et al. Central memory CD4+ $\mathrm{T}$ cells are responsible for the recombinant bacillus Calmette-Guérin äureC: hly vaccine's superior protection against tuberculosis. J Infect Dis 2014;210:1928-37. [PUBMED]

78. Premo C, Apolo AB, Agarwal PK, Citrin DE. Trimodality therapy in bladder cancer: Who, what, and when? Urol Clin North Am 2015;42:169-80, vii. [PUBMED]

79. Weiss C, Wolze C, Engehausen DG, Ott OJ, Krause FS, Schrott KM, et al. Radiochemotherapy after transurethral resection for high-risk $\mathrm{T} 1$ bladder cancer: An alternative to intravesical therapy or early cystectomy? J Clin Oncol 2006;24:231824. [PUBMED]

80. Vanpouille-Box C, Diamond JM, Pilones KA, Zavadil J, Babb JS, Formenti SC, et al. TGFâ is a master regulator of radiation therapy-induced antitumor immunity. Cancer Res 2015;75:223242. [PUBMED]

81. Levy A, Massard C, Soria JC, Deutsch E. Concurrent irradiation with the anti-programmed cell death ligand-1 immune checkpoint blocker durvalumab: Single centre subset analysis from a phase 1/2 trial. Eur J Cancer 2016;68:156-62. [PUBMED]

82. Robins DJ, Sui W, Matulay JT, Ghandour R, Anderson CB, DeCastro GJ, et al. Long-term survival outcomes with intravesical nanoparticle albumin-bound paclitaxel for recurrent nonmuscle-invasive bladder cancer after previous bacillus Calmette-Guérin therapy. Urology 2017;103:149-53. [PUBMED]

83. Shore ND, Boorjian SA, Canter DJ, Ogan K, Karsh LI, Downs TM, et al. Intravesical rAd-IFNá/Syn3 for patients with high-grade, bacillus CalmetteGuerin-refractory or relapsed non-muscleinvasive bladder cancer: A Phase II randomized study. J Clin Oncol 2017;35:3410-6. [PUBMED]
84. Zacchè MM, Srikrishna S, Cardozo L. Novel targeted bladder drug-delivery systems: A review. Res Rep Urol 2015;7:169-78.

85. Lu S, Neoh KG, Kang ET, Mahendran R, Chiong E. Mucoadhesive polyacrylamide nanogel as a potential hydrophobic drug carrier for intravesical bladder cancer therapy. Eur J Pharm Sci 2015;72:57-68. [PUBMED]

86. Lammers RJ, Witjes JA, Inman BA, Leibovitch I, Laufer M, Nativ $\mathrm{O}$, et al. The role of a combined regimen with intravesical chemotherapy and hyperthermia in the management of non-muscleinvasive bladder cancer: A systematic review. Eur Urol 2011;60:81-93. [PUBMED]

87. Juang T, Stauffer PR, Craciunescu OA, Maccarini PF, Yuan Y, Das SK, et al. Thermal dosimetry characteristics of deep regional heating of nonmuscle invasive bladder cancer. Int $\mathrm{J}$ Hyperthermia 2014;30:176-83. [PUBMED]

88. Arends TJ, Nativ O, Maffezzini M, de Cobelli O, Canepa G, Verweij F, et al. Results of a randomised controlled trial comparing intravesical chemohyperthermia with mitomycin $C$ versus bacillus calmette-guérin for adjuvant treatment of patients with intermediate- and high-risk nonmuscle-invasive bladder cancer. Eur Urol 2016;69:1046-52. [PUBMED]

89. Lammers RJM, Witjes WPJ, Hendricksen K, et al. Smoking status is a risk factor for recurrence after transurethral resection of non-muscle-invasive bladder cancer. Eur Urol. 2011;60:713720. [PubMed][Google Scholar]

90. Herr HW. The value of a second transurethral resection in evaluating patients with bladder tumors. J Urol.1999;162:74-76. [PubMed] [Google Scholar]

91. Sfakianos JP, Kim PH, Hakimi AA, Herr HW. The effect of restaging transurethral resection on recurrence and progression rates in patients with nonmuscle invasive bladder cancer treated with intravesical bacillus Calmette-Guerin. J Urol. 2014;191:341-345. [PMC free article] [PubMed] [Google Scholar]

92. Daneshmand S, Schuckman AK, Bochner BH, et al. Hexaminolevulinate blue-light cystoscopy in non-muscle-invasive bladder cancer: review of the clinical evidence and consensus statement on 
appropriate use in the USA. Nat Rev Urol. 2014;11:589-596. [PubMed] [Google Scholar]

93. Daneshmand S, Patel S, Lotan Y, et al. Efficacy and safety of blue light flexible cystoscopy with hexaminolevulinate in the surveillance of bladder cancer: a phase iii, comparative, multicenter study. J Urol.2018;199:1158-1165. [PubMed] [Google Scholar]

94. Lotan Y, Bivalacqua TJ, Downs T, et al. Blue light flexible cystoscopy with hexaminolevulinate in non-muscle-invasive bladder cancer: review of the clinical evidence and consensus statement on optimal use in the USA - update 2018. Nat Rev Urol. 2019;16:377-386. [PMC free article] [PubMed] [Google Scholar]

95. Chou R, Selph S, Buckley DI, et al. Comparative effectiveness of fluorescent versus white light cystoscopy for initial diagnosis or surveillance of bladder cancer on clinical outcomes: systematic review and meta-analysis. J Urol. 2017;197:548558. [PubMed] [Google Scholar]

96. Rink M, Babjuk M, Catto JW, et al. Hexyl aminolevulinate-guided fluorescence cystoscopy in the diagnosis and follow-up of patients with non-muscle-invasive bladder cancer: a critical review of the current literature. Eur Urol. 2013;64:624-638. [PubMed] [Google Scholar]

97. Naito S, Algaba F, Babjuk M, et al. The Clinical Research Office of the Endourological Society (CROES) multicentre randomised trial of narrow band imaging-assisted transurethral resection of bladder tumour (TURBT) versus conventional white light imaging-assisted TURBT in primary non-muscle-invasive bladder cancer patients: trial protocol and 1-year results. Eur Urol. 2016;70:506515. [PubMed][Google Scholar]

98. Chang SS, Boorjian SA, Chou R, et al. Diagnosis and treatment of non-muscle invasive bladder cancer: AUA/SUO Guideline. J Urol. 2016;196: 1021-1029. [PubMed] [Google Scholar]

99. Lotan Y, Black PC, Caba L, et al. Optimal trial design for studying urinary markers in bladder cancer: a collaborative review. Eur Urol Oncol. 2018;1:223-230. [PubMed] [Google Scholar]

100. Chou R, Gore JL, Buckley D, et al. Urinary biomarkers for diagnosis of bladder cancer: a systematic review and meta-analysis. Ann Intern
Med. 2015;163:922-931. [PubMed] [Google Scholar]

101. W B Gill, J L Huffman, E S Lyon, D H Bagley, H W Schoenberg, F H Straus 2nd Selective surface staining of bladder tumors by intravesical methylene blue with enhanced endoscopic identificationPMID: 6202385 DOI: 10.1002/10970142(19840615)53:12<2724::aid-cncr282053 1230>3.0.co;2-b

102. Muhammad Abdus Salam" Golam Maula Choudhury Surgical Technology \& Simulation: Instrumentation \& Technology III PD40-01 Effectiveness of endoscopic injection of $2 \%$ lignocaine in to the bladder wall to control obturator jerk during trans urethral resection of bladder tumor(TURBT)

103. Grossman HB, Messing E, Soloway M, et al. Detection of bladder cancer using a point-ofcare proteomic assay. JAMA. 2005;293:810-816. [PubMed] [Google Scholar]

104 Botteman MF, Pashos CL, Redaelli A, et al. The health economics of bladder cancer: a comprehensive review of the published literature. Pharmacoeconomics. 2003;21:13151330. [PubMed][Google Scholar]

105. Theodorescu D, Wittke S, Ross MM, et al. Discovery and validation of new protein biomarkers for urothelial cancer: a prospective analysis. Lancet Oncol. 2006;7:230-240. [PubMed] [Google

106 van Rhijn BW, van der Poel HG, van der Kwast TH Urine markers for bladder cancer surveillance: a systematic review. Eur Urol. 2005;47:736-748. [PubMed] [Google Scholar]

107. Ehdaie B, Theodorescu D. Molecular markers in transitional cell carcinoma of the bladder: new insights into mechanisms and prognosis. Indian J Urol. 2008;24:61-67. [PMC free article] [PubMed] [Google Scholar]

108. Theodorescu D. Molecular pathogenesis of urothelial bladder cancer. Histol Histopathol. 2003;18:259-274. [PubMed] [Google Scholar]

109. Gui Y, Guo G, Huang Y, et al. Frequent mutations of chromatin remodeling genes in transitional cell carcinoma of the bladder. Nat Genet. 2011;43:875878. [PMC free article] [PubMed][Google Scholar]

110. Guo G, Sun X, Chen C, et al. Whole-genome and whole-exome sequencing of bladder cancer identifies frequent alterations in genes involved in sister chromatid cohesion and segregation. Nat 
Genet. 2013;45:1459-1463. [PubMed] [Google Scholar]

111. Korkolopoulou P, Levidou G, Trigka EA, et al. A comprehensive immunohistochemical and molecular approach to the $\mathrm{PI} 3 \mathrm{~K} / \mathrm{AKT} / \mathrm{mTOR}$ (phosphoinositide 3-kinase/v-akt murine thymoma viral oncogene/ mammalian target of rapamycin) pathway in bladder urothelial carcinoma. BJUInt. 2012;110:E1237-E1248. [PubMed] [Google Scholar]

113 Knowles M.A., Hurst C.D. Molecular biology of bladder cancer: New insights into pathogenesis and clinical diversity. Nat. Rev. Cancer. 2015; 15:25-41. doi: 10.1038/nrc3817. [PubMed] [CrossRef][Google Scholar]

114. Martin-Doyle W., Kwiatkowski D.J. Molecular biology of bladder cancer. Hematol./Oncol. Clin. North. Am. 2015;29:191-203. doi: 10.1016/ j.hoc.2014.10.002. [PMC free article] [PubMed] [CrossRef][Google Scholar]

115. Richard S. Matulewicz, MD, MS ${ }^{1,2}$ and Gary D. Steinberg, $\mathrm{MD}^{1}$ Author information Copyright and License information Disclaimer, Nonmuscle-invasive Bladder Cancer: Overview and Contemporary Treatment Landscape of Neoadjuvant Chemoablative Therapies, Rev Urol. 2020; 22(2): 43-51.PMCID: PMC7393683, PMID: 32760227

116. Matulay JT, Soloway M, Witjes JA, et al. Riskadapted management of low-grade bladder tumors: recommendations from the International Bladder Cancer Group. BJU Int. 2020;125:497505.[PubMed] [Google Scholar]

117. Donat SM, North A, Dalbagni G, Herr HW. Efficacy of office fulguration for recurrent lowgrade papillary bladder tumors less than $0.5 \mathrm{~cm} . J$ Urol. 2004;171:636-639. [PubMed] [Google Scholar]

118. Hurle R, Lazzeri M, Vanni E, et al. Active surveillance for low risk nonmuscle invasive bladder cancer: a confirmatory and resource consumption study from the BIAS Project. J Urol.2018;199:401

119. Richard S. Matulewicz, MD, MS ${ }^{1,2}$ and Gary D. Steinberg, $\mathrm{MD}^{1}$ Non-muscle-invasive Bladder Cancer: Overview and Contemporary Treatment Landscape of Neoadjuvant Chemoablative Therapies, Rev Urol. 2020; 22(2): 43-51.

120. Shelley M, Court JB, Kynaston H, et al. Intravesical bacillus Calmette-Guérin in Ta and T1 bladder cancer. Cochrane Database Syst
Rev. 2000;2000(4):CD001986. [PMC free article] [PubMed][Google Scholar]

121. Shelley MD, Court JB, Kynaston H, et al. Intravesical bacillus Calmette-Guerin versus mitomycin $\mathrm{C}$ for $\mathrm{Ta}$ and $\mathrm{T} 1$ bladder cancer. Cochrane Database Syst Rev. 2003;2003(3): CD003231. [PubMed][Google Scholar]

122. Sylvester RJ, van der Meijden AP, Oosterlinck W, et al. Predicting recurrence and progression in individual patients with stage Ta T1 bladder cancer using EORTC risk tables: a combined analysis of 2596 patients from seven EORTC trials. Eur Urol. 2006;49:466-465. discussion 475477. [PubMed][Google Scholar]

123. Fernandez-Gomez J, Madero R, Solsona E, et al. Predicting nonmuscle invasive bladder cancer recurrence and progression in patients treated with bacillus Calmette-Guerin: the CUETO scoring model. J Urol. 2009;182:21952203. [PubMed] [Google Scholar]

124. Cambier S, Sylvester RJ, Collette L, et al. EORTC nomograms and risk groups for predicting recurrence, progression, and disease-specific and overall survival in non-muscle-invasive stage TaT1 urothelial bladder cancer patients treated with 1-3 years of maintenance bacillus CalmetteGuerin. Eur Urol. 2016;69:60-69.[PubMed] [Google Scholar]

125. Bruinsma SM, Bangma CH, Carroll PR, Leapman MS, Rannikko A, Petrides N, et al. Active surveillance for prostate cancer: a narrative review of clinical guidelines. Nat Rev Urol. 2016;13:151-167. [PubMed][Google Scholar]

126. Klotz L. Active surveillance and focal therapy for low-intermediate risk prostate cancer. Transl Androl Urol. 2015;4:342-354. [PMC free article] [PubMed] [Google Scholar]

127. Bahouth Z, Halachmi S, Meyer G, Avitan O, Moskovitz B, Nativ O. The natural history and predictors for intervention in patients with small renal mass undergoing active surveillance. Adv Urol. 2015;2015:692014. [PMC free article] [PubMed] [Google Scholar]

128 Ha SC, Zlomke HA, Cost N, Wilson S. The past, present, and future in management of small renal masses. J Oncol. 2015;2015:364807. [PMC free article] [PubMed] [Google Scholar]

129. Soloway MS, Bruck DS, Kim SS. Expectant management of small, recurrent, noninvasive papillary bladder tumors. J Urol. 2003;170(2 Pt 1):438-441. [PubMed] [Google Scholar] 